\title{
La resolución de problemas entre iguales: incidencia de la mediación del ordenador en los procesos de interacción y en el aprendizaje
}

\author{
MANOLI PIFARRÉ TURMO Y JAUME SANUY BURGUÉS
}

UNIVERSIDAD DE LLEIDA

\begin{abstract}
RESUMEN
En el presente trabajo se analiza la incidencia de la mediación del ordenador en la resolución de problemas y en los procesos de interacción entre parejas de alumnos de la Enseñanza Secundaria Obligatoria. Para ello, se diseña e implementa una propuesta de enseñanza-aprendizaje que guía el proceso de resolución de problemas e incide en los procesos de interacción entre iguales. Esta propuesta se realiza en dos modalidades o contextos de aprendizaje distintos: un contexto utiliza la mediación del ordenador y el otro contexto no.

El análisis de los resultados obtenidos en nuestro trabajo muestra la incidencia positiva de la mediación de la hoja de cálculo en el proceso de aprendizaje de los alumnos y en los procesos de interacción que presentan las distintas parejas durante la resolución de un problema. Los alumnos que han resuelto los problemas con la hoja de cálculo obtienen resultados estadísticamente superiores a los obtenidos por los alumnos que no han utilizado esta herramienta informática y presentan, principalmente, una interacción compartida.
\end{abstract}

Palabras clave: interacción entre iguales, resolución de problemas, procesos de enseñanzaaprendizaje mediados por ordenador.

\section{Peer problem solving: the incidence of computer mediation in interaction and learning processes}

In this work, the incidence of computer mediation in problem solving and in interactions processes among students of Secondary Education is analysed. We have designed and implemented a didactical sequence to improve dyadic interaction and problem solving strategies. This didactical sequence is carried out in two different learning contexts: a) using the computer mediation in order to solve the problems and b) not using the computer to do so.

Evaluation results showed the positive incidence when using spreadsheet mediation in interaction and learning processes of the students. The students that have used the spreadsheet to solve the problems show co-operative interaction and obtain better results than those who have not used this software.

Keywords: peer-group interaction, problem solving, computing mediation teaching-learning processes.

Agradecimientos: Este trabajo es parte de la Tesis Doctoral de la primera autora, dirigida por el segundo autor. Dicha investigación ha sido posible gracias a una ayuda para la financiación de proyectos de investigación de la Universidad de Lleida, proyecto $\mathrm{n}^{\circ} 812$. También queremos agradecer las aportaciones realizadas por el Dr. Angel Huguet a la redacción final de este artículo. 
Correspondencia con los autores: Departamento de Pedagogía y Psicología. Facultad de Ciencias de la Educación. Universidad de Lleida. Campus de la Caparrella, s/n. 25192 Lleida, pifarre@pip.udl.es

\section{EXTENDED SUMMARY}

The main objective of our work is to study the incidence in the learning of mathematical contents and in the quality of the dyads interaction. The next three variables have been studied in an instructional process with Secondary Education students: a) the individual attributes of the different members of the group; b) the organisation and the guidance of the students interaction processes with some questions about the problem and c) the mediation of the characteristics of an open software, as a spreadsheet, in the mathematical problem solving.

To reach this objective, we designed and implemented a didactical sequence to improve dyadic interaction processes with the guidance of the problem solving process. This guidance consist in a protocol with some questions and orientations about the problem to solve, this protocol is headed as "papers to think the problem".

The main hypothesis of our work is the external guidance on relevant aspects and strategies to solve the problem might have a positive incidence in the quality of interaction and in learning processes among dyads.

In order to analyse the incidence of the mediation of the computer in the students learning and interaction processes, the didactical sequence is carried out in two different learning contexts: one context use the computer mediation in order to solve the problems, particularly a spreadsheet, and the other context do not use the computer.

92 students of the third course of Secondary Education (13-14 years old) took part in this research. We gather data from two dependent variables: a) mathematical contents learning level and b) characteristics of dyads interaction, considering two independent variables: a) learning context and b) individual attributes of the dyads members (high homogeneous, heterogeneous, low homogeneous).

The results show the positive incidence of spreadsheet mediation in the students learning processes. Students who have used the spreadsheet to solve the problems obtained better results than the students that have not use this software. This result goes in the same direction as other studies showing the educational possibilities of computing tools for teaching.

The use of the computer has positive incidence in the peer interaction processes during the problem solving. The students that have used the spreadsheet to solve the problems show better co-operation behaviours, that is to say, a problem solving process in which the two members of the dyad have an active participation. On the other hand, the students who have not used the spreadsheet show a parallel interaction, both members of the dyad distribute the actions to solve the problem and carry out different simultaneous actions.

Our research also show that the attributes of the dyadic members exert an incidence of the learning level and in the interaction processes. From this point of view, the students who have worked in heterogeneous dyads have a great increase in their learning and present a shared interaction. If we attended to the definition of this category in our work, these dyads help to each other more frequently than the students who have worked in high and homogeneous dyads.

Although the difference that we have already mentioned, the interaction presented by the three types of dyads in the two learning contexts is mainly a shared interaction. Somehow, this 
demonstrates that the didactical sequence had a positive incidence in the dyads interaction processes and promoted a shared problem solving.

\section{RESUMEN EXTENSO}

El principal objetivo de nuestro trabajo es estudiar cómo inciden en el aprendizaje de contenidos matemáticos y en las características de la interacción entre iguales la incorporación de las tres variables siguientes en el diseño e implementación de un proceso instruccional con alumnos de ESO: a) las características individuales de los miembros del grupo; b) la organización y la estructuración de los procesos de interacción mediante la formulación de preguntas sobre el problema a resolver y c) la mediación de las características de un programa informático abierto, como es una hoja de cálculo, en la resolución de problemas matemáticos.

Para conseguir este objetivo, se diseña e implementa una propuesta de enseñanza-aprendizaje que pretende incidir en los procesos de interacción entre iguales durante la resolución de un problema matemático. Para ello, se diseña un protocolo formado por un conjunto de interrogantes y orientaciones que guían el proceso de resolución de un problema -y que denominamos "hojas para pensar el problema".

La principal hipótesis de nuestro trabajo es que la guía externa de las características de la interacción de las parejas hacia aspectos relevantes del proceso y de las estrategias de resolución del problema incidirá positivamente en la calidad de la interacción entre los dos alumnos y en el aprendizaje que se deriva.

Con el objetivo de analizar la incidencia que tiene en el aprendizaje de los alumnos y en los procesos de interacción la mediación de las características del ordenador durante la resolución de problemas matemáticos, la propuesta de enseñanza-aprendizaje se realiza en dos modalidades o contextos de aprendizaje distintos: un contexto que incorpora la mediación del ordenador, concretamente del programa informático de una hoja de cálculo, para resolver los problemas y el otro contexto que no utiliza la mediación del ordenador.

Se realiza un diseño experimental en el que participan un total de 92 alumnos de tercero de E.S.O. del Instituto "Ronda" de la ciudad de Lleida. Se recogen datos de dos variables dependientes-el nivel de aprendizaje de contenidos matemáticos y las características de la interacción de las parejas de alumnos-y en función de dos variables independientes -el contexto de aprendizaje y las características de los miembros que forman las distintas parejas de alumnos de nuestro estudio (homogéneas altas, homogéneas bajas y heterogéneas).

El análisis de los resultados obtenidos en nuestro trabajo muestra la incidencia positiva de la mediación de la hoja de cálculo en el proceso de aprendizaje de los alumnos. Los alumnos que han resuelto los problemas con la hoja de cálculo obtienen resultados estadísticamente superiores a los obtenidos por los alumnos que no han utilizado esta herramienta informática.

El uso del ordenador también incide positivamente en los procesos de interacción que presentan las distintas parejas durante la resolución de un problema. Los alumnos que han utilizado esta herramienta informática presentan, principalmente, una interacción compartida, es decir, un solo proceso de resolución en el cual los dos miembros de la pareja participan activamente. En cambio, el grupo de alumnos que no ha utilizado el ordenador presenta en mayor medida que el grupo "contexto ordenador" una interacción en paralelo, es decir, los dos miembros de la pareja se reparten las tareas para resolver el problema y realizan acciones diferentes y de manera simultánea.

Los resultados obtenidos en nuestro trabajo en función de las características de las parejas en que se han agrupado los alumnos muestran que las características del compañero con el que se trabaja influyen en el nivel de aprendizaje conseguido. En este sentido, destacamos la mejora en el aprendizaje que presentan los alumnos que han trabajado en las parejas heterogéneas, los cuales 
al finalizar el proceso instruccional obtienen resultados estadisticamente similares a los obtenidos por las parejas homogéneas altas.

A pesar de las diferencias observadas, el tipo de interacción que presentan los tres tipos de parejas en los dos contextos de aprendizaje es mayoritariamente una interacción compartida. Desde nuestro punto de vista, este resultado permite afirmar que la intervención educativa diseñada ha incidido positivamente en los procesos de interacción de los alumnos favoreciendo una resolución compartida de los problemas matemáticos.

\section{INTRODUCCIÓN}

Un gran número de investigaciones ha dedicado sus esfuerzos a estudiar la incidencia de los procesos de interacción entre iguales en la resolución de problemas. La principal hipótesis de trabajo que ha guiado la planificación de estos estudios es que la ayuda mutua entre los alumnos, así como la negociación de nuevos significados, el desarrollo de nuevas estrategias y la construcción de nuevo conocimiento repercuten positivamente en el aprendizaje (por ejemplo: Ellis y Gauvain, 1992; Rogoff, 1993; Lacasa y Herranz, 1995).

Esta argumentación guarda relación con la hipótesis de construcción social del conocimiento en la teoría de Vygotsky (1979). Desde esta perspectiva teórica se defiende que el aprendizaje y el desarrollo son fruto de la relación con otras personas, y con herramientas instrumentales y simbólicas aportadas por la cultura. Este axioma ha abonado el terreno para el desarrollo de investigaciones que analizan las características del aprendizaje cuando se resuelven tareas a partir de la interacción entre alumnos que trabajan con un ordenador.

Las características intrínsecas del ordenador permiten definirlo como una herramienta altamente interactiva que puede mediar en el desarrollo de procesos cognitivos superiores (Martí, 1992; Lajoie, 1993; Rodríguez Illera, 1997; Salomon y Perkins, 1998). Diferentes estudios muestran que en cierta medida las características generales del ordenador facilitan la interacción entre alumnos y propician un aprendizaje cooperativo. Diversos factores explican esta afirmación, entre los cuales destacamos los dos siguientes: en primer lugar, el feedback inmediato que aporta la pantalla del ordenador a una acción del alumno favorece la discusión entre los diferentes miembros del grupo (Nastasi, Clements y Battista, 1990; Nastasi y Clements, 1992). En segundo lugar, los alumnos deben hacer explícitas las órdenes que introducirán en el ordenador a fin de que éste realice las 
acciones apropiadas para resolver la tarea; este hecho permite que los otros miembros del grupo conozcan el proceso de pensamiento de su compañero y puedan así, debatirlo o expresar su acuerdo (Weir, 1989; Kanselaar et al., 2001).

A pesar de los potenciales beneficios que, para la mejora del aprendizaje en los alumnos, suponen los procesos de interacción entre iguales y la mediación del ordenador en estos procesos; los trabajos de revisión y de meta-análisis a los que se han sometido las investigaciones que versan sobre este tema sólo han mostrado correlaciones parciales e inconsistentes entre los procesos de interacción entre iguales -con o sin la mediación del ordenador- y el aprendizaje que se deriva de éstos (Webb, Troper y Fall, 1995; Susman, 1998).

Entre las explicaciones a la diversidad de los resultados educativos obtenidos con el uso del ordenador, destaca el contexto de enseñanza-aprendizaje cuyas características son especialmente relevantes como variable explicativa de la eficacia de la herramienta informática en el aprendizaje de los alumnos (Salomon, Perkins y Globerson, 1991; Martí, 1992). Las características del contexto educativo inciden en el desarrollo de determinados procesos cognitivos, metacognitivos y de interacción social implicados en la actividad de los alumnos con el ordenador y, por tanto, influyen en los resultados educativos que se consiguen. Las conclusiones de los trabajos realizados con programas abiertos de simulación (Duffy y Barowy, 1995) o de hipertexto (Kommers y Lanzing, 1998) ilustran esta afirmación, ya que estos estudios muestran que si no se enseñan a los alumnos estrategias específicas para trabajar y resolver las tareas con el programa informático, los alumnos que utilizan el ordenador pueden obtener resultados inferiores a los que obtienen los alumnos que no lo utilizan.

Por ello, el primer objetivo que nos planteamos en este artículo es el de revisar las principales variables del contexto educativo que inciden en los procesos de interacción y de aprendizaje entre iguales mediados por ordenador. El segundo objetivo de este trabajo es analizar la incidencia del diseño de un proceso de enseñanza-aprendizaje concreto en el aprendizaje de contenidos 
matemáticos, así como en las características de la interacción entre iguales; todo ello llevado a cabo en un contexto natural de aula con alumnos de $3^{\circ}$ de ESO. El diseño del proceso de enseñanzaaprendizaje que analizamos en este artículo ha incorporado dos variables del contexto educativo que trata la bibliografía revisada. Ambas variables favorecen la interacción entre iguales y el aprendizaje que se deriva de ellos. Estas dos variables son: i) la organización y la estructuración de los procesos de interacción mediante la formulación de preguntas sobre el problema a resolver y ii) la mediación de las características de un programa informático abierto, como es una hoja de cálculo, en la resolución de problemas matemáticos.

En los párrafos siguientes expondremos las principales conclusiones teóricas formuladas en la bibliografía existente sobre la influencia de las características del contexto educativo en el aprendizaje. Dichas conclusiones dan cuenta de las variables que explican el aprendizaje obtenido en la interacción entre iguales mediada por ordenador. La bibliografía consultada proporciona un gran número de variables, que hemos agrupado en las tres variables siguientes de carácter más general: a) las variables externas que inciden en los procesos interactivos, considerando como variables externas: las características de los individuos, del grupo y de las formas de trabajo que estos individuos presentan durante el proceso de resolución (McAuliffe y Dembo, 1994; Lacasa y Herranz, 1995); b) las características específicas de la interacción entre iguales y el procedimiento para diseñar procesos instruccionales que enseñen a los alumnos a interactuar de manera positiva y aprender en grupo (Cohen, 1994; Webb, Troper y Fall, 1995; King, 1994, 1997); y c) las características del programa informático específico que se utiliza (Mevarech, Silber y Fine, 1991; Susman, 1998).

\section{a) La incidencia de las características de los miembros del grupo en los procesos de interacción}

Entre las características de los individuos que inciden en el aprendizaje entre iguales, a partir de los objetivos de nuestro estudio, destacamos el nivel de habilidad cognitiva que presentan los miembros del grupo. Un conjunto de trabajos analizan cómo influye el nivel cognitivo de los alumnos en los 
procesos de interacción entre iguales y en el aprendizaje; para ello estos trabajos clasifican los grupos de alumnos en homogéneos y heterogéneos, en función del nivel de habilidad de los miembros del grupo (Forman, 1992, McAuliffe y Dembo, 1994).

Las conclusiones más destacables a las que llegan estos estudios son, en primer lugar, el hecho de que el trabajo de alumnos con diferente nivel de habilidad en la resolución de una tarea dinamiza los procesos de interacción entre los miembros del grupo. En segundo lugar, la constatación de que en los grupos homogéneos medios y heterogéneos, el nivel de ayuda entre los miembros del grupo es mayor que en los grupos homogéneos bajos y altos. Y, en tercer lugar, la evidencia de que los grupos heterogéneos formados por alumnos con una variación en el nivel de habilidad cognitiva moderada son los grupos que obtienen mejores rendimientos.

b) La incidencia de las características de la situación de enseñanza-aprendizaje en los procesos de interacción

Los estudios que analizan las características específicas de los procesos interactivos que se desarrollan en situaciones de aprendizaje entre iguales señalan que distintos tipos de interacción promueven también un aprendizaje diferente, tanto desde el punto de vista cuantitativo como cualitativo (Webb y colaboradores 1989, 1994, 1995; King, 1991, 1997; Cohen, 1994; entre otros). En esta línea de trabajo, el estudio de Roselli, Gimelli y Hechan (1995) tiene como principal objetivo reconocer las consecuencias que podría tener el tipo de interacción en la cognición individual. Después de un período experimental en el que los alumnos habían trabajado en parejas, se analiza el rendimiento individual en la resolución de problemas. Se categorizan los procesos de interacción de los alumnos en tres modalidades básicas, definidas a partir de diferentes grados de interacción socio-cognitiva: egocéntrica, asimétrica y simétrica e igualitaria. Estos autores llegan a la de que el éxito individual está asociado a la interacción igualitaria; y que, por el contrario, los resultados individuales menos exitosos se encuentran en los alumnos que han tenido una interacción egocéntrica con la pareja. 
Las conclusiones de estos estudios han propiciado la realización de diferentes trabajos de investigación que diseñan diferentes situaciones educativas, cuyos objetivos inciden en los procesos de interacción entre iguales para favorecer un mejor aprendizaje. Hemos agrupado los trabajos revisados en tres grupos o líneas de investigación. En primer lugar, los estudios que diseñan procesos instructivos orientados a enseñar estrategias de comunicación a los alumnos, de forma que estas estrategias potencien un trabajo en grupo más efectivo. (Hooper, Temiyakarn y Williams, 1993; Webb, Troper y Fall, 1995). Principalmente, este grupo de estudios enseña a los alumnos estrategias de análisis de una demanda, de síntesis y de parafraseo.

En segundo lugar, se encuentran los estudios que asignan roles o funciones concretas a los alumnos durante la resolución conjunta de una tarea. Estos trabajos se basan en el hecho de que los miembros de un grupo asumen de manera implícita o explícita un rol o un estatus en el proceso de resolución de la tarea. Los diferentes roles asumidos por los miembros del grupo no siempre tienen una incidencia positiva en el aprendizaje (Cohen, 1994).

Y, en tercer lugar, se incluyen los estudios que modifican los procesos de interacción a partir de la realización de preguntas sobre el proceso de resolución del problema o tarea (King, 1991, 1994, 1997; O’Donnell y Dansereau, 1992; Shaw, 1997). Estos estudios persiguen dos objetivos. El primer objetivo consiste en la organización y estructuración de los procesos de interacción; la formulación de preguntas y respuestas sobre el proceso de resolución del problema entre los miembros del grupo favorece que la interacción de éstos verse sobre aspectos relevantes de la tarea y de su resolución, aspectos que no aparecen espontáneamente en todos los grupos de iguales y que pueden repercutir positivamente en el aprendizaje de los alumnos.

El segundo objetivo que estos estudios pretenden llegar a alcanzar es el aprendizaje explícito de estrategias de resolución de problemas por parte de los alumnos, ya que la respuesta a las preguntas que se plantean implican, bien que el alumno se está cuestionando aspectos del proceso de resolución, o bien que el alumno ha ejecutado diferentes procedimientos con el objetivo de resolver 
con éxito el problema. En este sentido, un gran número de investigaciones realizadas desde el ámbito educativo de las estrategias de aprendizaje propone, como una metodología de enseñanza de estrategias, el diseño de diversos tipos de pautas u hojas escritas. En estas pautas se recopilan los principales interrogantes que el alumno debe formularse para alcanzar el objetivo propuesto en la tarea educativa, así como las acciones más importantes que éste debe realizar para resolver la tarea planteada -por ejemplo, en el campo de la lectura, Solé (1993); en el campo de la escritura, Castelló (1995); y en el campo de las matemáticas Schoenfeld (1985), King (1991) y Barberà (1995).

c) La incidencia de las características de la mediación del ordenador en los procesos de interacción

Finalmente, el tercer factor que explica las diferencias en el nivel de aprendizaje alcanzado por los alumnos en la resolución tareas en grupo mediante el uso de un ordenador, se refiere a las características específicas del programa informático que se utiliza. Susman (1998), en un metaanálisis realizado sobre 23 estudios que analizan situaciones de aprendizaje cooperativo mediado por ordenador, concluye que el nivel de aprendizaje obtenido y las características de la interacción entre alumnos están relacionados con el tipo de programa informático utilizado. En este sentido, los estudios que utilizan programas de tipo tutorial o programas de resolución de problemas obtienen mejores resultados que los estudios que utilizan programas de tipo ejercitación y práctica. La explicación a estos resultados se encuentra en el hecho de que el primer tipo de programas favorece el aprendizaje cooperativo porque implica la resolución de problemas más complejos, amplios y poco definidos, en los cuales el grupo de alumnos puede explorar y discutir diferentes estrategias de resolución; en cambio, en los programas de ejercitación y práctica se plantean problemas muy definidos que exigen la aplicación de una única estrategia para su resolución (Mevarech y Light, 1992).

En conclusión, si bien la mediación del ordenador no garantiza un entorno cooperativo, la elección o el diseño de un determinado programa informático propicia el trabajo y la interacción en grupo, 
proporcionando apoyo a aspectos como la reflexión y el control de las acciones que realizan los alumnos, la exploración de diferentes perspectivas y maneras de solucionar la tarea, y la posibilidad de usar diferentes recursos (representaciones gráficas, consulta a diferente tipo de información) (Hooper, 1992; Susman, 1998).

\section{OBJETIVOS DEL TRABAJO EMPÍRICO}

Nuestro trabajo se plantea dos objetivos:

1. El primer objetivo pretende analizar la incidencia que tiene en el aprendizaje de los alumnos y en los procesos de interacción la mediación de las características del ordenador en la resolución de problemas matemáticos.

Para alcanzar este objetivo en nuestro trabajo, en primer lugar, se ha diseñado un proceso instruccional que utiliza los procesos de interacción entre iguales como instrumento para el aprendizaje de procedimientos de resolución de problemas. La propuesta de enseñanza-aprendizaje analizada en este artículo, que recoge las conclusiones de los trabajos realizados por King (1991, 1994, 1997) y Schoenfeld, (1985, 1992), ha elaborado un protocolo formado por un conjunto de interrogantes y orientaciones que guían el proceso de resolución de un problema -y que denominamos "hojas para pensar el problema". Los alumnos resuelven un problema en parejas y responden conjuntamente a las diferentes cuestiones que se plantean en el protocolo, el cual se plantea como una guía externa de la interacción de las parejas de alumnos hacia aspectos relevantes del proceso y de las estrategias de resolución del problema. Esta guía sirve al propósito de aumentar la calidad de la interacción entre los dos alumnos, así como de incrementar el aprendizaje que se deriva de dicha interacción.

En segundo lugar, la propuesta de enseñanza-aprendizaje se ha llevado a cabo en dos modalidades o contextos de aprendizaje distintos: un contexto que incorpora la mediación del ordenador, concretamente del programa informático de una hoja de cálculo para resolver los problemas, y otro 
contexto que no utiliza la mediación del ordenador. Así, en ambos contextos de aprendizaje, las características de la propuesta didáctica son las mismas (objetivos, contenidos, actividades, materiales didácticos y estrategias de enseñanza utilizadas por el profesor), la única característica diferente es la mediación del programa de una hoja de cálculo.

En nuestro estudio se ha seleccionado el programa informático de una hoja de cálculo porque se trata de un programa abierto que ofrece múltiples posibilidades de uso por parte de los alumnos, entre las que destacamos la manipulación sencilla de entornos numéricos; la formulación de hipótesis matemáticas (qué pasa si... se modifica un número o una fórmula de la hoja de trabajo) y la representación gráfica de la notación matemática. Creemos que estas características, acompañadas del tipo de problemas que se plantean en la propuesta de enseñanza-aprendizaje problemas amplios, de una complejidad elevada y cuya resolución permite la aplicación de diferentes procedimientos y estrategias-, pueden favorecer una interacción colaborativa entre las parejas de alumnos.

2. El segundo objetivo de nuestro trabajo consiste en analizar el nivel de aprendizaje de contenidos matemáticos alcanzado por los alumnos y el tipo de interacción que se presenta entre éstos, en función de las características de los miembros que forman las distintas parejas de alumnos de nuestro estudio. La muestra de alumnos se ha agrupado en virtud de su rendimiento inicial en el área de las matemáticas en tres tipos de parejas: homogéneas altas, heterogéneas y homogéneas bajas.

\section{MÉTODO}

\section{Muestra y diseño experimental}

La muestra que participa en nuestro estudio está formada por cuatro clases naturales de alumnos de tercero de E.S.O. del Instituto "Ronda" de la ciudad de Lleida. La muestra está formada por un total de 92 alumnos que se distribuyen en función de dos variables independientes (cuadro 1): 
- Variable independiente 1: contexto de aprendizaje. Los alumnos se distribuyen en dos grupos: a) el grupo "contexto ordenador", formado por 46 alumnos que utilizan el programa informático como instrumento mediador en el aprendizaje de contenidos matemáticos y en la interacción entre las parejas; b) el grupo "contexto no ordenador", formado por 46 alumnos que no se sirven del ordenador como mediador del aprendizaje; a los alumnos de este grupo se les brinda la oportunidad de usar una calculadora para resolver los problemas. Se ha denominado a este segundo grupo "no ordenador", y no se ha contemplado el uso de la calculadora porque, por un lado, nuestro estudio no ha llevado a cabo un tratamiento educativo especial del uso de la calculadora como instrumento mediador en la resolución de problemas, tan sólo se permite su uso. Por otro lado, los problemas planteados en la propuesta didáctica contienen un gran número de datos, con el objetivo de equiparar a los dos grupos ante la dificultad de cálculo numérico de los problemas que deben resolver, se permite el uso de la calculadora en el grupo de alumnos "no ordenador".

- Variable independiente 2: tipo de pareja. Tal y como se muestra en el cuadro 1, en cada uno de los dos contextos de aprendizaje, los alumnos se distribuyen en tres grupos: A) "homogéneo alta", formado por 20 alumnos con un rendimiento inicial alto en el contenido matemático de la proporcionalidad -10 alumnos en cada contexto; B) homogéneo baja, formado por 36 alumnos con un rendimiento inicial bajo en el contenido matemático de la proporcionalidad -18 alumnos en cada contexto; y C) heterogéneo, formado por 36 alumnos, los cuales se distribuyen de la siguiente manera: 18 alumnos con un rendimiento inicial alto en el contenido matemático de la proporcionalidad y 18 alumnos con un rendimiento bajo.

Se ha considerado que un alumno de rendimiento inicial bajo, es aquel que ha obtenido una puntuación igual o inferior a 0,5 puntos en la resolución individual de los cinco problemas de la prueba pre-test. Se ha estimado que un alumno de rendimiento inicial alto, es aquel que ha obtenido una puntuación igual o superior a 1 punto en la resolución de estos cinco problemas. 
Son dos los profesores que instruyen a la muestra de alumnos. Nuestro estudio no ha contemplado al profesor como sujeto de la investigación, pero somos conscientes de que su actuación y sus características concretas influyen en el aprendizaje de los alumnos, por este motivo se han llevado a cabo las dos actuaciones siguientes: en primer lugar, el diseño y la planificación de los métodos comunes de enseñanza que va a emplear el profesorado; en segundo lugar, la distribución equilibrada de los grupos de alumnos por docente, de modo que los dos profesores que han participado en el estudio ha instruido a un grupo de alumnos del contexto "ordenador" y a un grupo de alumnos del contexto "no ordenador".

Insertar cuadro 1

\section{Procedimiento}

El procedimiento experimental consta de tres fases:

La primera fase ha consistido en la realización de siete problemas sobre el contenido matemático de la proporcionalidad, que forman la prueba de evaluación inicial o pre-test. Cinco de los problemas deben resolverse individualmente y los otros dos deben resolverse en pareja.

La segunda fase o fase de intervención corresponde a la realización por parte de los alumnos de una propuesta de enseñanza-aprendizaje de procedimientos de resolución de problemas sobre el contenido de la proporcionalidad durante un trimestre de clase (30 horas de clase, aproximadamente).

En líneas generales, la propuesta de enseñanza-aprendizaje que se aplica a los dos grupos de alumnos (contexto ordenador y contexto no ordenador) se divide en dos partes bien diferenciadas. Una primera parte tiene como principal objetivo el aprendizaje del contenido de la proporcionalidad directa. Una segunda parte favorece el aprendizaje colaborativo de estrategias de resolución de problemas complejos sobre proporcionalidad. En esta segunda parte, las parejas de alumnos resuelven 10 problemas contextualizados en la vida cotidiana con la ayuda de un material didáctico 
que denominamos "hojas para pensar el problema".

Este material didáctico tiene como principal objetivo guiar y enriquecer el proceso de resolución del problema. Se plantea a los alumnos diferentes interrogantes, indicaciones y sugerencias sobre los posibles procedimientos que deben emplear para resolver el problema. De este modo, este material didáctico ha sido diseñado para constituir una ayuda externa que las parejas de alumnos utilizan mientras resuelven el problema.

La guía induce a los alumnos a reflexionar sobre la necesidad de aprender y utilizar las cinco estrategias generales que siguen los expertos en resolución de problemas, según las investigaciones realizadas en este campo (por ejemplo: Krulik y Rudnick, 1989; Schoenfeld, 1985, 1992). Las cinco estrategias generales trabajadas en la guía son: a) entender y analizar el problema; b) planificar un plan de resolución; c) organizar los datos y el plan de resolución en un cuadro de doble entrada, d) resolver el problema, y e) evaluar el proceso de resolución del problema y el resultado obtenido. Cada estrategia define un objetivo general que el alumno puede alcanzar si realiza diferentes procedimientos en función de las características del problema. Cada estrategia se acompaña de un icono que resume e ilustra el objetivo general a conseguir en cada momento. En el cuadro 2 se proporciona un ejemplo de las indicaciones presentadas a los alumnos para evaluar el proceso de resolución del problema y el resultado obtenido.

------- Insertar cuadro 2 ------

Tal y como hemos señalado anteriormente, dado que nuestro trabajo empírico no ha considerado al profesor como sujeto de la investigación, en la propuesta didáctica se han diseñado las características de los procesos de enseñanza que éste debe tener en cuenta. Con el objetivo de incidir en los procesos de interacción y de aprendizaje de estrategias de resolución de problemas, el profesor ha utilizado los tres métodos que se detallan a continuación, y que se consignan en la amplia literatura dedicada al estudio sobre cómo enseñar estrategias para resolver problemas (por ejemplo: Schoenfeld, 1985; Delclos y Harrington, 1991; King, 1991, 1994; ): 
a) Instrucción directa: el profesor presenta los diferentes procedimientos que se trabajan en la guía.

b) Instrucción guiada: el profesor modela cómo utilizar la guía como instrumento de ayuda para pensar y resolver el problema en parejas.

c) Análisis y discusión metacognitiva del proceso de resolución del problema. Por un lado, el profesor dinamiza el trabajo de las parejas supervisando su proceso de resolución y realiza diferentes preguntas y orientaciones que pueden dirigir o reorientar la resolución del problema. Por otro lado, y al finalizar la resolución de cada problema, una pareja de alumnos del grupo expone los principales procedimientos utilizados para resolver el problema, mientras que el resto del grupo analiza y valora el proceso y el producto obtenido.

La tercera fase del proceso experimental ha consistido en la resolución de los problemas que forman la prueba de evaluación final o post-test. Los siete problemas de la prueba post-test presentan características similares a los resueltos en la prueba de evaluación inicial o pre-test.

\section{Unidades de análisis e instrumentos de medida}

En nuestro estudio, se aplican dos instrumentos de análisis que recogen datos sobre dos variables dependientes: el rendimiento en la resolución de problemas y las características de la interacción de los alumnos durante la resolución de un problema.

\section{Evaluación del rendimiento o el resultado en la resolución de problemas}

La evaluación de esta variable se realiza mediante dos pruebas escritas, una pre-test y una post-test, que constan de siete problemas cada una. Cinco problemas se resuelven individualmente y dos, en pareja.

Con el objetivo de evaluar la incidencia del uso de la hoja de cálculo en la resolución de problemas y en los procesos de interacción entre los dos miembros de la pareja, los alumnos del grupo “contexto ordenador" resuelven dos problemas de la prueba de evaluación post-test mediante el uso 
del ordenador, uno que deben resolver individualmente y otro en pareja. Los alumnos del grupo "contexto no ordenador" resuelven estos dos problemas de la prueba post-test con una calculadora que les facilita las operaciones de cálculo.

La corrección de las pruebas pre-test y post-test se ha realizado siguiendo una pauta en la que se detallan los criterios de evaluación de cada problema. Cada problema se puntúa de 0 a 1 . Para garantizar la fiabilidad de la pauta de corrección y de la evaluación de esta variable dependiente, el $25 \%$ de las pruebas han sido corregidas por dos jueces. El análisis estadístico de los resultados obtenidos por los dos jueces se ha realizado mediante la prueba estadística de Correlación de Pearson. La fiabilidad entre los dos jueces es muy alta, las correlaciones se sitúan entre .86 y 1.00 .

\section{Evaluación de las características de la interacción de los alumnos durante la resolución de un problema}

Esta variable dependiente pretende analizar cómo las características específicas de cada contexto de aprendizaje -ordenador/no ordenador- modifica los procesos de interacción social de los alumnos durante la resolución de problemas matemáticos.

Los datos de análisis de esta variable se han recogido durante la resolución de un problema en pareja de la prueba post-test. Los alumnos del grupo "contexto ordenador" resuelven el problema mediante el uso de la hoja de cálculo, mientras que el grupo de alumnos "contexto no ordenador" lo resuelve con una calculadora.

Los datos sobre las características de la interacción de las parejas de alumnos se recogen a partir de la grabación en vídeo del proceso de resolución del problema, con y sin uso de la hoja de cálculo de 12 parejas de alumnos escogidas al azar entre el total de la muestra. Las parejas se distribuyen en 6 parejas pertenecientes a cada contexto, de las cuales dos son homogéneas altas, dos heterogéneas y dos, homogéneas bajas. Posteriormente, la información grabada se transcribe en unos protocolos y, finalmente, se categoriza en función de las categorías diseñadas en nuestro estudio. El proceso de 
categorización se ha realizado utilizando un procedimiento de toma de decisiones por consenso entre dos jueces.

Nuestro estudio ha seguido los trabajos de Forman (1992), Lacasa y Herranz (1995), y Roselli, Gimelli y Hechan (1995) para diseñar las categorías de análisis de nuestro instrumento de evaluación y estudiar la manera en que las parejas de alumnos interactúan durante la resolución de un problema. En estos trabajos se destaca la importancia de la variable: "manera en que los iguales interactúan durante la resolución de una tarea" para explicar el aprendizaje de los alumnos. En nuestro trabajo se han definido las tres categorías siguientes:

- Interacción compartida: Los dos miembros de la pareja participan activamente y se implican por igual en la resolución del problema. Los alumnos presentan un solo proceso de resolución. Por ejemplo se observan las siguientes acciones: un alumno que propone y otro que ejecuta; un alumno que dirige las acciones de su compañero; y un alumno que propone comentarios, indicaciones, sugerencias, preguntas... sobre las acciones que ejecuta su compañero.

- Interacción paralela: Los dos miembros de la pareja participan activamente en la resolución del problema, pero realizan acciones diferentes de manera simultánea. Se observan bien contactos puntuales entre los dos alumnos (comentarios, miradas...) o bien acciones de un miembro que el otro toma en cuenta (sugerencias, repartición de tareas...). Se observan dos procesos de resolución.

- No interacción: La actividad no es compartida en ningún sentido.

\section{RESULTADOS}

Organizaremos los resultados obtenidos en las dos variables dependientes de nuestro estudio: resultado del aprendizaje y características de la interacción, en función de los dos objetivos que nos proponíamos al inicio del proceso experimental.

a) Contexto de aprendizaje: ordenador/no ordenador 
El análisis de la varianza ANOVA de dos factores (contexto x tipo de pareja) aplicado a las puntuaciones obtenidas por los alumnos en la prueba de evaluación final o post-test, tomando las puntuaciones obtenidas en la prueba pre-test como covariante, muestra diferencias estadísticamente significativas en función de la variable independiente "contexto de aprendizaje" $[\mathrm{F}(1,80)=7.496$, $\mathrm{p}<.01]$

El análisis de las medias obtenidas por los alumnos en función del "contexto de aprendizaje" revela que el rendimiento en la resolución de problemas de la prueba post-test es significativamente mejor entre los alumnos que han utilizado el ordenador $[\mathrm{t}(1,45)=2.371, \mathrm{p}<.05]$, tal y como se puede observar en la figura 1.

-Insertar figura 1

El estudio de las características de la interacción de los alumnos durante la resolución de un problema en pareja en los dos contextos de aprendizaje de nuestro trabajo contribuye a explicar en detalle el rendimiento obtenido por los alumnos.

Como se muestra en la figura 2, se observan notables diferencias en la manera de interactuar de los alumnos durante la resolución de un problema en los dos contextos de aprendizaje. Los alumnos del contexto ordenador presentan una interacción compartida en la práctica totalidad del tiempo utilizado para la resolución del problema (98.82\%); en cambio, el grupo de alumnos que no utiliza el ordenador muestra este tipo de interacción en un $69 \%$ del tiempo total empleado. Esta diferencia es estadísticamente significativa $[\mathrm{t}(1,23)=2.913, \mathrm{p}<.05]$.

Insertar figura 2

Cabe reseñar diferencias importantes que se observan en el tipo de interacción en paralelo. En este caso, el grupo de alumnos que no utiliza el ordenador presenta en mayor medida este tipo de interacción, en un $20.04 \%$, frente al $0.53 \%$ del grupo de alumnos del contexto ordenador. 
Atendiendo al tamaño de la muestra, también consideramos esta diferencia estadísticamente significativa $[\mathrm{t}(1,23)=-2.201, \mathrm{p}=.05]$.

\section{b) Tipo de pareja: Homogénea alta, homogénea baja y heterogénea}

El análisis de la varianza también muestra diferencias en función del tipo de pareja en la que han trabajado los alumnos, diferencias que, atendiendo al tamaño de la muestra, podemos considerar estadísticamente significativas $[\mathrm{F}(2,80)=2.378, \mathrm{p}=.099]$. La prueba de contrastes de Games Howell apunta diferencias significativas, por un lado, entre las parejas homogéneas bajas y las homogéneas altas y, por otro lado, entre las parejas homogéneas bajas y las heterogéneas. En cambio, no se observan diferencias significativas entre las parejas heterogéneas y las homogéneas altas (figura 1).

El análisis de las puntuaciones medias obtenidas por los alumnos en función "tipo de pareja" señala, por un lado, que los alumnos que han trabajado en parejas homogéneas bajas obtienen resultados estadísticamente inferiores a los que han participado en parejas heterogéneas $[\mathrm{t}(1,71)=-3.682, \mathrm{p}<$. 0001], y a los alumnos que han trabajado en parejas homogéneas altas $[\mathrm{t}(1,55)=-3.721, \mathrm{p}<.0001]$. Por otro lado, no se observan diferencias significativas entre los alumnos que han formado las parejas homogéneas altas y los alumnos que han hecho lo propio en parejas heterogéneas.

En nuestro trabajo también se han observado diferencias en el tipo de interacción presente en los diferentes tipos de parejas, aunque éstas no son estadísticamente significativas. Como se observa en la figura 3, las parejas heterogéneas se aprecia en mayor medida una interacción compartida. En cambio, las parejas homogéneas altas, presentan en mayor medida una interacción en paralelo. Finalmente, subrayamos el hecho que las parejas homogéneas bajas no registran ningún tipo de interacción en un 15\% del tiempo utilizado para resolver el problema. 


\section{DISCUSIÓN Y CONCLUSIONES}

El análisis de los resultados obtenidos en nuestro trabajo revela la incidencia positiva de la mediación de la hoja de cálculo en el proceso de aprendizaje de los alumnos. Los alumnos que han resuelto los problemas con la hoja de cálculo obtienen resultados estadísticamente superiores a los alcanzados por los alumnos que no se han servido de esta herramienta informática. Este resultado es muy similar al que arrojan diversos estudios en los que se destacan las potencialidades educativas de la herramienta informática. Entre los estudios más cercanos a nuestro trabajo, presentamos, en primer lugar, los resultados conseguidos por Lambrecht (1993), que utiliza un programa de hoja de cálculo para resolver problemas sobre el contenido de la proporcionalidad, en el campo temático de la banca. En este estudio, los alumnos que utilizan el ordenador ostentan niveles de aprendizaje estadísticamente superiores a los conseguidos por los alumnos de un grupo control que utilizan la calculadora para aprender y resolver el mismo tipo de problemas.

En segundo lugar, Gómez-Granell et al. (1997) obtienen también resultados superiores en el aprendizaje de contenidos sobre proporcionalidad cuando se utiliza un programa informático, diseñado específicamente para aprender este tipo de contenidos, respecto a los resultados de los alumnos de un grupo control que no utiliza el ordenador.

En nuestro trabajo, el uso del ordenador también ha incidido positivamente en los procesos de interacción que presentan las distintas parejas durante la resolución de un problema. Los alumnos que han utilizado esta herramienta informática registran, principalmente, una interacción compartida, es decir, un solo proceso de resolución en el cual los dos miembros de la pareja participan activamente. En cambio, en el grupo de alumnos que no ha utilizado el ordenador se aprecia una interacción en paralelo en mayor medida que el grupo "contexto ordenador", es decir, los dos miembros de la pareja se reparten las tareas para resolver el problema y realizan acciones diferentes de manera simultánea. Estos resultados coinciden con los obtenidos por otros estudios que han analizado el tipo de interacción que se da entre los alumnos cuando resuelven diferentes 
tareas con el uso del ordenador (Nastasi y Clements, 1992; Amigues y Agostinelli, 1992; Steren, 1996; entre otros).

Diferentes argumentos explican este hecho. En primer lugar, trabajar con una única pantalla de ordenador en la cual aparecen todas las acciones que realiza el alumno favorece la implicación de los dos miembros de la pareja y la discusión sobre las acciones a realizar para conseguir los objetivos planteados en la tarea, de modo que se potencia una resolución más compartida y colaborativa (O’Malley, 1992; Griffin, Belyaeva y Soldatova, 1992; Dillenbourg y Self, 1992). Esta explicación ha sido corroborada con el uso de diferentes programas informáticos como, por ejemplo, el Logo (Nastasi, Clements y Battista, 1990), o la escritura de un texto mediante un procesador de textos (Ferrari, Caviglia y Degl'innocenti, 1992).

En segundo lugar, la pantalla del ordenador desempeña la función de puente de unión entre los diferentes niveles de representación de la tarea y del proceso para resolverla. En la pantalla se genera una nueva representación de la tarea que los alumnos pueden manipular con la ayuda del teclado y el ratón, favoreciendo así la interacción compartida entre ellos (Amigues y Agostinelli, 1992).

Los resultados obtenidos en nuestro trabajo, en función de las características de las parejas en que se han agrupado los alumnos, revelan que las características del compañero con el que se trabaja influyen en el nivel de aprendizaje obtenido. En este sentido, destacamos la mejora del aprendizaje en las parejas heterogéneas, cuyos miembros, al finalizar el proceso instructivo obtienen resultados estadísticamente similares a los alcanzados por las parejas homogéneas altas.

Estos resultados siguen la misma tendencia destacada en otros estudios realizados desde esta perspectiva, en los cuales se defiende la hipótesis consistente en que la resolución de una tarea en la que se interactúa con un compañero de un nivel de habilidad diferente potencia un aprendizaje más efectivo que la interacción con un compañero de un nivel de habilidad similar (Rogoff, 1993; Forman, 1992; Weeb y colaboradores, 1991, 1994, 1995; Steren, 1996). 
Entre las conclusiones de este conjunto de investigaciones, se destacan las que se refieren a diferencias importantes en la cantidad y el tipo de ayuda que se facilitan los alumnos de las parejas en virtud de sus características. Las parejas heterogéneas presentan un índice de ayuda mutua mayor que las parejas homogéneas, tanto las de nivel alto como las de nivel bajo.

Estas diferencias en la manera de interactuar que se aprecian en los alumnos durante la resolución de una tarea, en función de las características del compañero con el que se trabaja, también se consignan en nuestro trabajo. Las parejas heterogéneas registran en mayor medida una interacción compartida, y atendiendo a la definición de esta categoría en nuestro estudio, presentan una mayor frecuencia de ayuda mutua que las parejas homogéneas altas y bajas.

A pesar de las diferencias observadas, la interacción que presentan las tres clases de parejas en los dos contextos de aprendizaje es mayoritariamente colaborativa. Desde nuestro punto de vista, este resultado permite afirmar que la intervención educativa diseñada -concretada en el material didáctico "hojas para pensar el problema"- ha incidido positivamente en los procesos de interacción de los alumnos, y ha favorecido, al mismo tiempo, la resolución compartida de los problemas matemáticos.

En conclusión, y a partir de los principales objetivos planteados en nuestro trabajo, señalamos tres perspectivas futuras en la investigación sobre las variables educativas que favorecen tanto en el aprendizaje entre iguales como en las posibilidades educativas del ordenador.

En primer lugar, nuestro estudio ha señalado la posibilidad de diseñar y aplicar un proceso de enseñanza-aprendizaje en un contexto natural de aula que incida positivamente en los procesos de interacción entre iguales y en el aprendizaje que se deriva de ellos. En este sentido, se han revelado eficaces tanto el diseño de la metodología empleada por el profesor, como la creación de una situación de enseñanza-aprendizaje, la cual se encarga de guiar el proceso de resolución de una tarea mediante la formulación y la respuesta a cuestiones relevantes que afectan a dicho proceso. La guía del proceso de resolución adopta la forma del material didáctico llamado "hojas para pensar el 
problema". Ahora bien, estamos de acuerdo con King (1997) en que es necesario diseñar estudios que permitan conocer nuevos datos sobre cómo enseñar a los alumnos a formular y a responder autónomamente diferentes tipos de preguntas relevantes que les permitan resolver el problema sin seguir un protocolo previamente establecido.

En segundo lugar, nuestro trabajo ha mostrado que la mediación de las características educativas del ordenador puede potenciar y mejorar la interacción y el aprendizaje entre iguales. Siguiendo las conclusiones expresadas por Susman (1998), creemos que es necesario continuar con el estudio de las variables educativas que pueden ayudar a introducir de manera más efectiva el uso del ordenador en los procesos de enseñanza-aprendizaje. Entre estas variables, cabe señalar el diseño y la utilización de programas informáticos específicos que potencien la resolución de una tarea de manera compartida; programas que den soporte, por ejemplo, a la reflexión por parte del grupo o a la exploración de diferentes materiales y de información.

En tercer lugar, nuestro estudio ha constatado efectos positivos que se dan tanto en los procesos de interacción entre iguales como en el nivel de aprendizaje en los alumnos agrupados de forma heterogénea. En este sentido, los resultados obtenidos en nuestro estudio siguen la misma tendencia destacada en otros trabajos de investigación que adoptan esta perspectiva. Desde estos trabajos, si bien se muestra que los procesos de dar y de recibir ayuda son distintos en función de las características de los miembros de la pareja, también se destaca la necesidad de conocer las condiciones en que el proceso de dar y de recibir ayuda es más efectivo; es decir, cuándo y en qué condiciones, el alumno, a partir de la ayuda recibida, construye una representación que mejora la resolución de una tarea. Así mismo, estos estudios se plantean la necesidad de crear situaciones de enseñanza-aprendizaje que potencien el desarrollo de estas condiciones.

\section{BIBLIOGRAFIA}


AMIGUES, R. y AGOSTINELLI, S. (1992). Collaborative problem-solving with a computer: how can an interactive learning environment be designed? European Journal of Psychology of Education, VII (4), 325-337.

BARBERÀ, E. (1995). Estrategias en matemáticas. Cuadernos de Pedagogía, 237, 29-32.

CASTELLÓ, M. (1995). Estrategias para escribir pensando. Cuadernos de Pedagogía, 237, 22-28.

COHEN, E. G. (1994). Restructuring the classroom: Conditions for productive small groups. Review of Educational Research, 64, 1-35.

DELCLOS, V. y HARRINGTON, C. (1991). Effects of strategy monitoring and proactive instruction on children's problem solving performance. Journal of Educational Psychology, 83 (1), 35-42.

DILLENBOURG, P. y SELF, J. A. (1992). A computational approach to socially distributed cognition. European Journal of Psychology of Education, VII (4), 353-372.

DUFFY, M. y BAROWY, W. (1995). Effects of constructivism and computer-facilitated strategies on achievement in heterogeneous secondary biology. Comunicación presentada a the annual meeting of the National Association for Research in science teaching, $68^{\text {th }}$, San Francisco, CA.

ELLIS, S. y GAUVAIN, M. (1992). Social and cultural influences on children's collaborative interactions. En L. T. Winegar y J. Valsiner (Eds.). Children's Development Within Social Context. Vol. 2: Research and methodology, (pàg. 155-180). Hillsdale, New Jersey: Lawrence Erlbaum Associates.

FERRARIS, M., CAVIGLIA, F. y DEGL'INNOCENTI, R. (1992). WordProf: A writing environment on computer. ETTI, 27 (1), 33-42.

FORMAN, E. A. (1992). Discourse, intersubjetivity, and the development of peer collaboration: A vigotskian approach. En L. T. Winegar y J. Valsiner (Eds.). Children's development within social context, vol. 1 (143-159). New Jersey: Lawrence Erlbaum Associates.

GÓMEZ-GRANELL, C., MARTÍ, E., GARCÍA-MILÀ, M. y STEREN, B. (1997). Un entorno informático interactivo integrado en el currículum de ciencias y matemáticas de ESO. Cultura y Educación, 6/7, 133156.

GRIFFIN, P.; BELYAEVA, A. y SOLDATOVA, G. (1992). Socio-historical concepts applied to observations of computer use. European Journal of Psychology of Education, VII (4), 269-286.

HOOPER, S. (1992). Effects of peer interaction during computer-based mathematics instruction. Journal of Educational Research, 85 (3), 180-189.

HOOPER, S.; TEMIYAKARN, C. y WILLIAMS, M. D. (1993). The effects of cooperative learning and learner control on high and average ability students. Educational Technology Research and Development, 41 (2), 5-18.

KANSELAAR, G., ERKENS, G., JASPERS, J. y SCHIJF, H. (2001). Computer supported collaborative learning. Teaching and Teacher Education 17, 123-129.

KING, A. (1991). Effects of training in strategic questioning on children's problem-solving performance. Journal of Educational Psychology, 83 (3), 307-317.

KING, A. (1994). Guiding knowledge construction in the classroom: Effects on teaching children how to question and how to explain. American Educational Research Journal, 30, 338-368.

KING, A. (1997). Ask to think-tel why: a model of transactive peer tutoring for scaffolding higher level complex learning. Educational Psychologist, 32 (4), 221-235.

KOMMERS, P. y LANZING, J. (1998). Mapas conceptuales para el diseño de sistemas hipermedia: navegación por la Web y autoevaluación. En C. Vizcarro y J. León. Nuevas tecnologías para el aprendizaje (103-128). Madrid: Pirámide

KRULIK, S. y RUDNICK, J. A. (1989). Problem solving: a handbook for senior high school teachers. Boston: Allyn and Bacon.

LACASA, P. y HERRANZ, P. (1995). Aprendiendo a aprender: resolver problemas entre iguales. Madrid: Ministerio de Educación y Ciencia. CIDE. 
LAJOIE, S. P. (1993). Computer environments as cognitive tools for enhancing learning. En S. P. Lajoie y S.

J. Derry (Eds.). Computers as Cognitive Tools, pp. 261-288.

LAMBRECHT, J. (1993). Applications software as cognitive enhancers. Journal of Research on Computing in Education, 25 (4), 506-520.

MARTÍ, E. (1992). Aprender con ordenadores en la escuela. Baracelona: ICE/Horsori.

MCAULIFFE, T. J. y DEMBO, M. J. (1994). Status rules of behavior in scenarios of peer learning. Journal of Educational Psychology, 86, 163-172.

MEVARECH, Z. R. y LIGHT, P. H. (1992). Peer-Based Interaction at the Computer: Looking backward, looking forward. Learning and Instruction, 2, 275-280.

MEVARECH, Z. R., SILBER, O. y FINE, D. (1991). Learning with computers in small groups: Cognitive and affective disorders. Journal of Educational Computing Research, 7 (2), 233-243.

NASTASI, B. y CLEMENTS, D. H. (1992). Social-cognitive behaviors and higher-order thinking in educational computer environments. Learning and instruction, 2, 215-238.

NASTASI, B., CLEMENTS, D. H. y BATTISTA, M. (1990). Social cognitive interactions, motivation and cognitive growth in Logo programming and CAI problem solving environments. Journal of Educational Psychology, 82, 150-158.

O'DONNELL, A. M. y DANSERAU, D. F. (1992). Scripted Cooperation in Student Dyad: A method for analyzing and Enhancing academic learning and performance. En R. Hertz-Lazarowitz y N. Miller (Eds.). Interaction in Cooperative Groups, pp. 120-141. Cambridge: University Press.

O'MALLEY, C. (1992). Designing computer systems to support peer learning. European Journal of Psychology of Education VII (4), 339-352.

RODRIGUEZ ILLERA, J.L. (1997). El aprendizaje mediado con ordenadores: realidades textuales y zona de desarrollo próximo. Cultura y Educación, 6/7, 77-90.

ROGOFF, B. (1993). Aprendices del pensamiento. El desarrollo cognitivo en el contexto social. Barcelona: Paidós (original publicado en inglés, 1990).

ROSELLI, N. D., GIMELLI, L. E. y HECHAN, M. E. (1995). Modalidades de interacción sociocognitiva en el aprendizaje de conocimientos en pareja. En P. F. Berrocal y M. A. M. Zabal (Comp.). La interacción social en contextos educativos (137-166). Madrid: Siglo XXI.

SALOMON, G., PERKINS, D. N. y GLOBERSON, T. (1991). Partners in cognition: extending human intelligence with intelligent technologies. Educational Researcher, 20 (3), 2-9.

SALOMON, G. y PERKINS, D.(1998). Individual and social aspects of learning. Review of Research in Education, 23, 1-24.

SCHOENFELD, A. (1985). Mathematical problem solving, New York: Academic Press.

SCHOENFELD, A. (1992). Learning to think mathematically: Problem Solving, Metacognition, and sense Making in mathematics. En D. Grows. Handbook for research on mathematics teaching and learning (334-370). New York: Macmillan Publishing Company.

SHAW, J. (1997). Cooperative problem solving: using K-W-D-L as an organizational technique. Teaching children mathematics, 3 (9), 482-486.

SOLÉ, I. (1993). Estrategias de lectura. Barcelona: Graó.

STEREN, B. (1996). Interacción entre iguales y procesos mediatizados por ordenador. Analisis e intervención en contexto escolar. Tesi doctoral no publicada. Universitat de Barcelona.

SUSMAN, E. B. (1998). Cooperative learning: a review of factors that increase the effectiveness of cooperative computer-based instruction. Journal of Educational Computing Research, 18 (4), 303-332.

VYGOTSKY, L. S. (1979). El desarrollo de los procesos psicológicos superiores. Barcelona: Grijalbo.

WEBB, N. (1989). Peer interaction and learning in small groups. Journal of Educational Research, 13 (1) 21-39. 
WEBB, N. M. y FARIVAR, S. (1994). Promoting helping behaviour in cooperative small groups in middle school mathematics. American Educational Research Journal, 31, 369-395.

WEBB, N. M., TROPER, J. D. y FALL, R. (1995). Constructive activity and learning in collaborative small groups. Journal of Educational Psychology, 87 (3), 406-423.

WEIR, S. (1989). Visions for the use of computers in classroom instruction. Harvard Educational Review, $59,61-73$. 
Manoli Pifarré Turmo

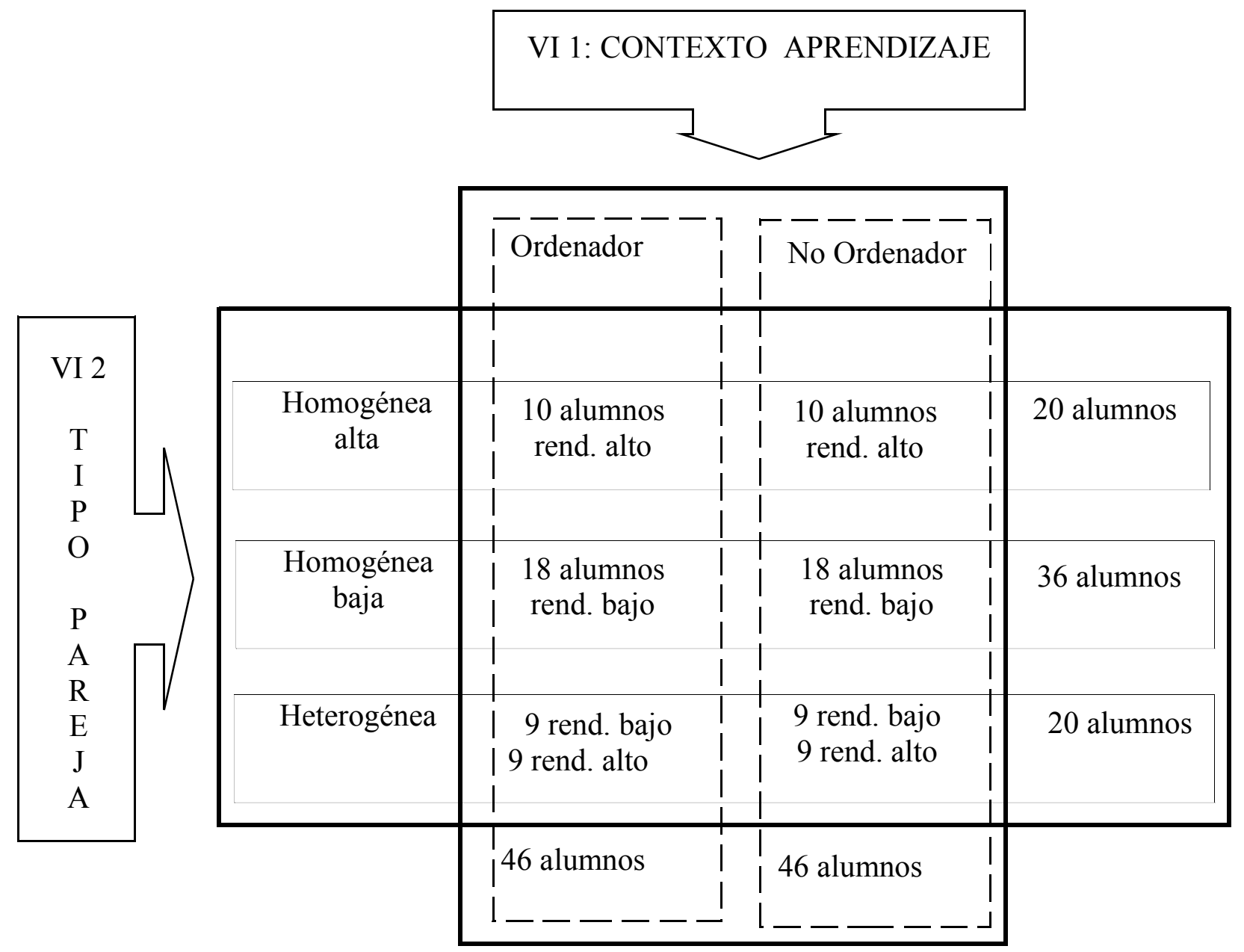

Cuadro 1: Distribución de la muestra de alumnos. (rend. = rendimiento). 


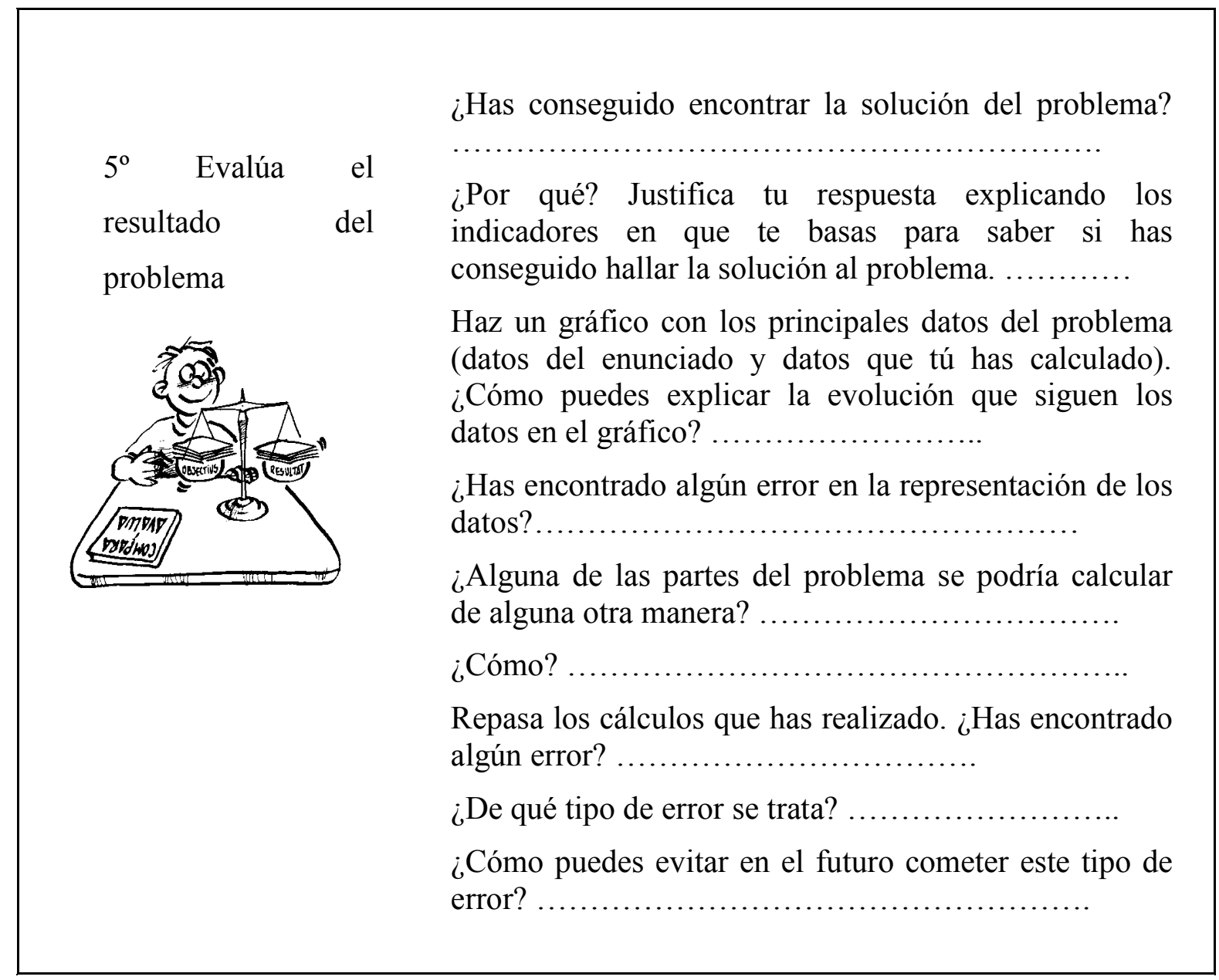

Cuadro 2: Ejemplo de las indicaciones para evaluar el resultado y el proceso de resolución del problema del material didáctico "hojas para pensar el problema". 
Manoli Pifarré Turmo

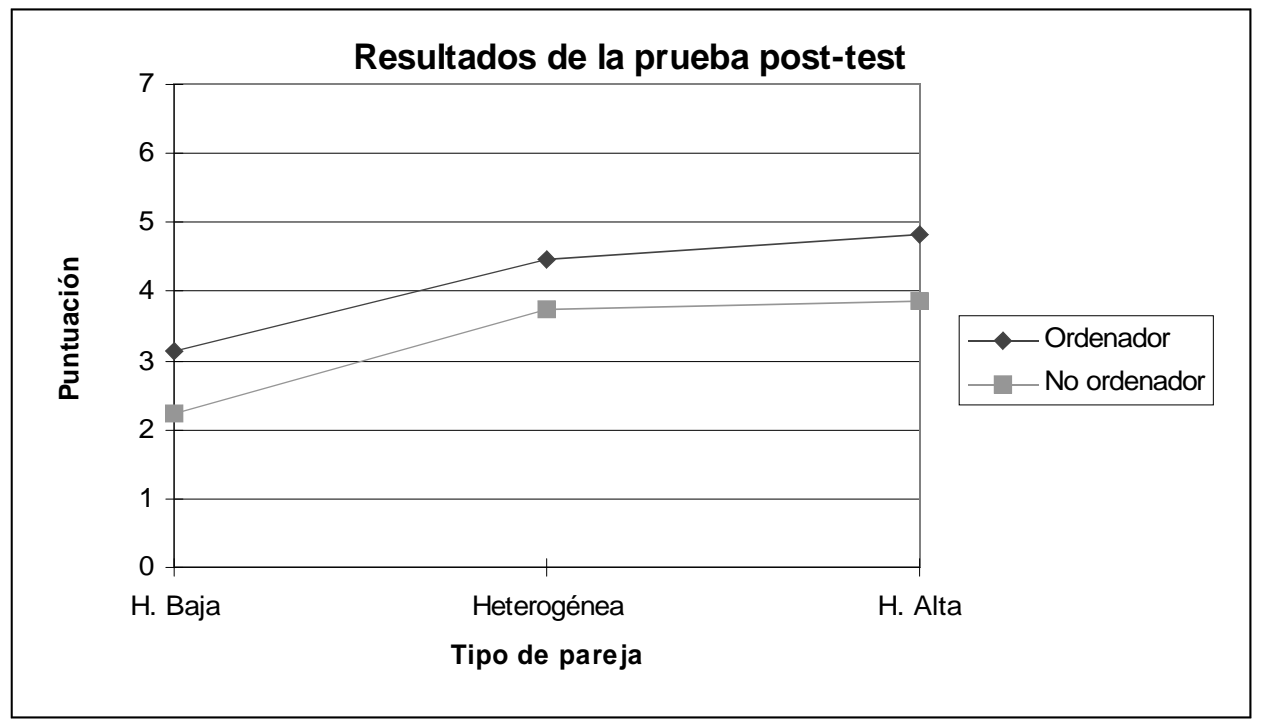

Figura 1. Resultados obtenidos en la prueba post-test en función de las variables independientes: contexto de aprendizaje y tipo de pareja.

- Insertar la figura 1 en la página 19 de este documento escrito 
Manoli Pifarré Turmo

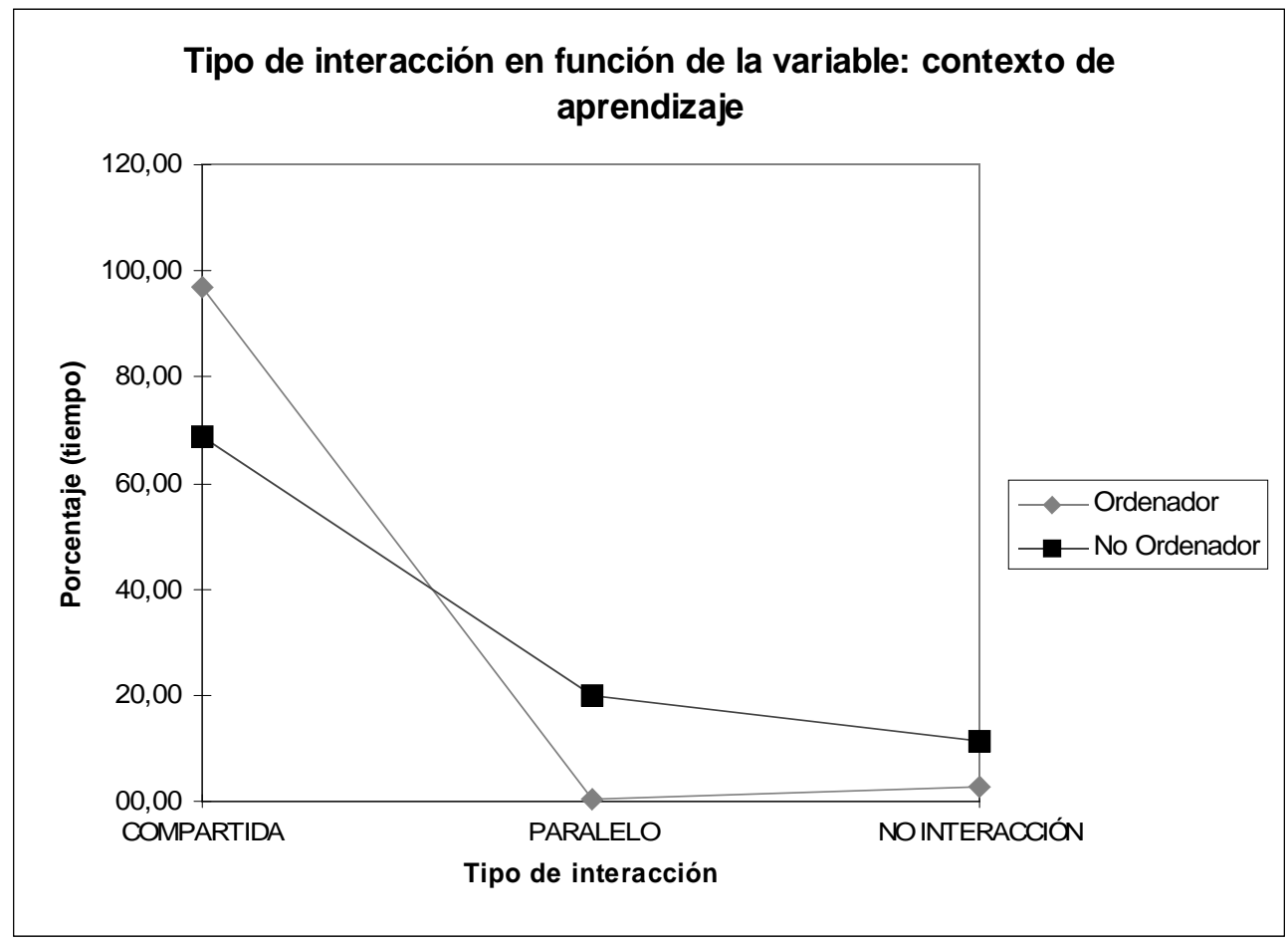

Figura 2. Características del tipo de interacción en función de la variable independiente: contexto de aprendizaje.

Insertar la figura 2 en la página 19 de este documento escrito 
Manoli Pifarré Turmo

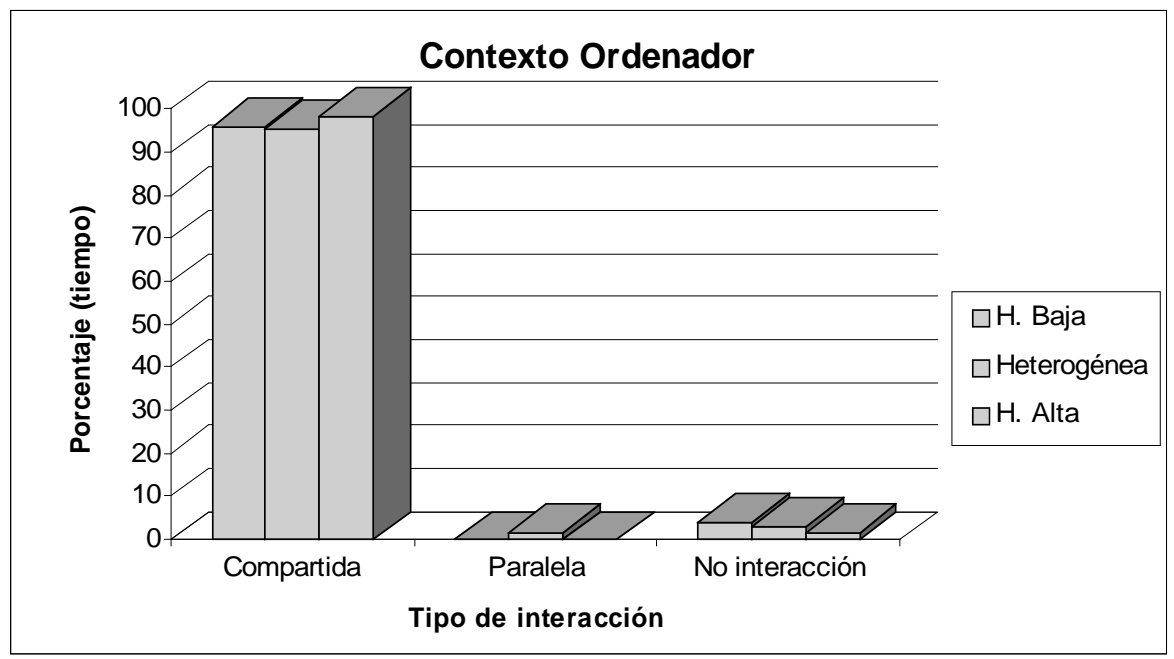

Figura 3. Características del tipo de interacción en función de la variable independiente: tipo de pareja.

Insertar la figura 3 en la página 20 de este documento escrito 